\title{
The changing face of neurosurgery for the older person
}

\author{
Ellie Edlmann ${ }^{1,2}$ () Peter C. Whitfield ${ }^{2}$
}

Received: 6 February 2020 / Revised: 17 April 2020 / Accepted: 18 April 2020 / Published online: 25 April 2020

(c) Springer-Verlag GmbH Germany, part of Springer Nature 2020

\begin{abstract}
Increased life expectancy and illness prevention and treatment have led to a growing population of older patients. These changes in patient population are apparent in neurosurgery; however, relatively little is reported about specific outcomes and prognostication in this group. This review summarises the challenges and management changes occurring in the treatment of three common neurosurgical pathologies; aneurysmal subarachnoid haemorrhage, head injury, and haemorrhagic stroke. A move towards less invasive neurosurgical techniques has implications on the risk-benefit profile of interventions. This creates the opportunity to intervene in older patients with greater co-morbidity, as long as improved outcomes can be evidenced. A critical part of assessing appropriateness for surgical intervention in older patients may be to change from a mindset of age to one of frailty and growing interest in scales assessing this may aid treatment decisions in the future.
\end{abstract}

Keywords Elderly $\cdot$ Frailty $\cdot$ Neurosurgery $\cdot$ Subarachnoid haemorrhage $\cdot$ Trauma

\section{Introduction}

Increased life expectancy, lower operative morbidities, and enhanced expectations from patients and their families has led to an increasing population of older patients with pathology that may be amenable to neurosurgical treatment.

In 2010, Chibbaro showed that the proportion of patients aged 70 and over admitted to a Parisian neurosurgical unit increased from $11 \%$ in 1983 to $25 \%$ in 2007 [6]. Surgical interventions also increased from $77 \%$ to $93 \%$ in this group, with a particular rise in brain tumour surgery. Such trends are set to continue and a recent report from a UK regional neurosurgical centre showed continued increases in elderly admissions from 2000 to 2013, particularly in emergency conditions such as traumatic head injury and spontaneous subarachnoid haemorrhage [42].

An important driver of increased admissions is the perceived improvement in outcomes in older patients. Chibbaro

Ellie Edlmann

eedlmann@nhs.net

1 Faculty of Health: Medicine, Dentistry and Human Sciences, University of Plymouth, N14, ITTC Building, Plymouth Science Park, Plymouth PL6 8BX, UK

2 Southwest Neurosurgical Centre, University Hospitals Plymouth NHS Trust, Derriford Road, Plymouth PL6 8DH, UK showed a dramatic drop in the immediate mortality rate, from 12 to $0.3 \%$ in older patients [6]. Shifting treatment algorithms, such as endovascular coiling rather than aneurysm clipping, account, in part, for improved outcomes. However, poor outcomes still prevail in older patients with emergency presentations: Whitehouse reported 5-year mortality of around 55\% in neurosurgical patients over 65 admitted as an emergency compared with less than $20 \%$ in elective patients [42]. Mortality is particularly increased within 6 months of neurosurgical treatment and, therefore, surgical approaches and their associated complications need to be carefully considered to improve outcomes.

In this review, we consider changes in practice and current treatment outcomes in older patients with aneurysmal subarachnoid haemorrhage, traumatic head injury, and haemorrhagic strokes. We focus on the assessment of frailty in older patients and how this can help to inform future treatment planning.

\section{Aneurysmal subarachnoid haemorrhage}

Incidental, unruptured cerebral aneurysms pose a risk of rupture over time; factors including size and location influence this risk [43]. It is logical, that as people live longer, there is a greater lifetime risk of incidental aneurysm rupture and presentation with a subarachnoid haemorrhage (SAH). 
Conversely, the first detection of an incidental cerebral aneurysm in later life means that there is a reduced effect of any preventative treatment due to the deceased remaining life expectancy in the elderly. In addition, the outcomes, in terms of quality of life and mortality, from treated aneurysmal SAH in older patients are variable. As such, it is important that we have robust data on all these aspects, to guide the management of both ruptured and unruptured aneurysms in the older person.

In 2006, Nieuwkamp et al. reported outcomes in 170 patients aged $\geq 75$ years presenting with aneurysmal SAH, where only $20 \%$ of patients were clipped and $8 \%$ coiled [27]. Overall, half the patients died and only one in six returned to independent function by discharge. Of their 60 patients admitted in a poor condition (those with a Glasgow Coma Score of 12 or less), none were independent at discharge. The strongest predictor of poor outcome in good-grade admissions was re-bleeding. Compared to younger SAH patients, those aged 75 and over were more likely to be female (perhaps due to greater life expectancy) and suffer with medical complications and hydrocephalus. The authors suggested that the early treatment of patients in a good condition may prevent re-bleeding and thus improve outcomes.

In 2013, Scholler analysed 265 patients over the age of 60 with aneurysmal SAH, with $36 \%$ aged between 70 and 79 and only $10 \%$ over 80 [33]. Challenges identified in this older population included increased co-morbidities ( $92 \%$ had at least one), the use of anti-thrombotic drugs (in 29\%) and increased prevalence of hydrocephalus requiring an EVD in those aged 70-80 years (77\%) compared to 60-70-year-olds $(61 \%)$. Interventional treatment was undertaken in $85 \%$ of cases, with clipping in $48 \%$ and coiling in $37 \%$, the latter more commonly in those aged over 80 . Outcome was clearly associated with age; death or poor outcome at discharge occurred in $73 \%$ of over 70 years old compared with $51 \%$ of 60-70 years old. Condition at presentation was also important with no poor grade at presentation patients (WFNS 4-5) aged over 80 experiencing a good outcome at discharge. It is important to note that although $70 \%$ of conservatively managed patients died, none were due to re-bleed, suggesting that it is the nature of the initial bleed and not any failure to treat, that results in the poor outcome.

There has also been a significant shift in practice in the last 15 years towards coiling, rather than clipping since the International Subarachnoid Aneurysm Trial (ISAT) [25]. Subsequent sub-group analysis of patients aged 65 years and over suggested a trend towards better outcomes with endovascular treatment compared to clipping, although this was subject to aneurysm location [30]. Lower rates of infectious and pulmonary complications and epilepsy occurred in the endovascular group. This was supported by the Barrow Ruptured Aneurysm Trial (BRAT) which showed significantly poorer outcomes at 1 year in patients over 50 years old treated with clipping [21]. A recent systematic review of endovascular treatment of ruptured aneurysms in patients aged over 65 reported good outcomes in $66 \%$, with a mortality rate of around $26 \%$ at 1 year [36]. With an $86 \%$ complete or near-complete occlusion rate at long-term follow-up, endovascular treatment is preferred to clipping and probably reduces risks for older patients.

Koffijberg analysed the cost-effectiveness of treating ruptured aneurysms in patients aged over 70 , identifying key parameters including patient age (and thus life expectancy), good or poor clinical condition on presentation, conservative or occlusive treatment (clipping or coiling) and good or poor outcomes [18]. Perhaps surprisingly, occlusive treatment of aneurysms translated into a health benefit for all older patients presenting within 4 days of $\mathrm{SAH}$, regardless of the good or poor condition at presentation. A later presentation, particularly 10 days or more after $\mathrm{SAH}$, was more likely to result in no or minimal treatment benefits. However, occlusive treatment was only found to be cost-effective in women aged 70-79, and men aged 70-74 presenting in a good condition, within 4 days of SAH. Thus, although potentially offering some clinical benefit, occlusive treatment is clearly more costly than conservative management and may be particularly difficult to justify in those aged 80 years and over presenting in a poor condition. It is, however, also important to highlight that studies assessing interventions such as this will contain inherent selection bias, by virtue of the fact that the patients undergoing intervention differ from those that were not. Such results should be treated with caution until clear, prospective, evidence is available.

It is apparent that patients presenting in a poor condition and aged over 70 are likely to have a poor outcome, and a conservative approach may be appropriate in these patients. More prospective research is needed to understand the risk-benefit profile of treating well patients, particularly those aged over 80 . The current literature supports active intervention for those under 80, with an awareness of the increased risk of complications, but more data are needed specifically in relation to outcomes with endovascular intervention.

\section{Head injury}

The vast majority of older patients admitted to a neurosurgical unit with head injury have a subdural haematoma, most commonly chronic subdural haematoma (CSDH), followed by mixed and then acute subdural haematoma (ASDH) [41].

A CSDH is a condition almost exclusively confined to the elderly, with a median age of 77 in the UK [4]. The post-traumatic pathophysiology is complex; it takes weeks to months for the collection of blood and fluid to expand: evidences support the theory that this is, at least in part, due 
to an escalating inflammatory process [8]. Many patients have no recollection of significant trauma and presentation is usually with cognitive impairment, gait disturbance, limb weakness, or headache. The mainstay of treatment has been surgical drainage $[4,31]$. However, recent trials have focused on medical treatments, such as steroids, for controlling the inflammatory response in $\mathrm{CSDH}$, and, therefore, either reducing CSDH recurrence following surgery or even as a first-line treatment $[9,15,19,24]$. Any treatment which reduces CSDH recurrence has the potential to reduce mortality, which can be as high as $18 \%$ at 6 months [31]. The outcome of these steroid trials is still awaited, but a move to more conservative treatments of CSDH could benefit older patients with multiple co-morbidities who are at increased risk from anaesthesia and surgical treatment.

ASDHs are traditionally considered to occur in higher impact trauma, leading to coma and hence a poorer prognosis, particularly in the elderly. However, due to co-existent cerebral atrophy in older patients, lower energy forces (falls) cause most traumatic brain injuries in this age group, and the onset of neurological deterioration secondary to an ASDH may be delayed. This provides an opportunity for definitive management, following an early CT scan, as recommended by NICE guidelines [26]. ASDH following a minor fall is often exacerbated by the fact that nearly two-third of these patients are on anti-thrombotic medications [20]. The increased use of these medications and an aging population are probably contributing to growing rates of ASDH in the elderly. A recent review of 165 ASDHs in a German hospital reported $41.2 \%$ of them occurring in patients aged 80 or over [44]. Importantly, although $90 \%$ of the patients aged $\geq 80$ years of age underwent surgery, only $24 \%$ experienced a favourable outcome at discharge (Glasgow outcome scale $4-5$ ), increasing to $36 \%$ at 3 -months. The mortality rate was high, $48 \%$ at 3 months, and predictors of an unfavourable outcome included $>5$ co-morbidities, GCS $\leq 8$ at admission and 24 hours, re-bleeding, and pneumonia. The use of anti-thrombotic medications at presentation is also clearly a risk factor for poor outcome in all patients [45]. Another series reported no survivors in patients with an ASDH aged over 90 and only one survivor out of 8 aged over 65 presenting with a GCS of less than 10 [41]. A recent systematic review of ASDH in the elderly reported on only seven eligible studies with the mean patient age ranging from 73 to 85 years [10]. Outcomes varied with a mortality rate ranging from 27 to $70 \%$, and a good functional outcome in $10-41 \%$ but with a presenting GCS $\leq 8$ representing a poor prognostic factor. However, overall, the studies were classified as low quality and were particularly lacking in assessments of patient frailty which may aid future research in this field. It is clear that poor neurology from the outset is associated with poor outcome; however, as the incidence of this pathology appears to be increasing in older patients, effective surgical decision-making tools are needed. In patients over 80 years old, with multi-morbidity and poor neurology, caution should be applied; however, it could be advocated that in all other circumstances surgical treatment should at least be considered. Attempts have been made at producing scoring systems to aid prognostication in elderly patients with ASDH, but more widespread validation of this is required [1].

It is notable that several large-scale studies assessing interventions for severe traumatic brain injury (TBI) in general, including decompressive craniectomy [7, 16], ICP monitoring [5], and cooling [2], have either excluded older patients or had minimal numbers of them. Whilst this makes understanding treatment in this age group challenging, it may also just be a reflection of the reality that fewer older patients are considered likely to survive intervention. This is supported by collaborations such as IMPACT (International Mission for Prognosis and Analysis of Clinical Trials in TBI) and CRASH (Corticosteroid Randomisation After Significant Head injury), who have used available evidence to develop prognostic calculators for TBI, where age is a corestratifying component and significantly increases chances of a poor outcome [17, 38]. Overall, it is recognised that a lack of evidence has led to varying practices and understanding about interventions for TBI in older patients, but it is clear that functional and cognitive recovery is significantly worse in this age group [13]. Understanding patient baseline function and morbidity is important, but recognising the high chance of a poor outcome in this age group often leads clinicians to follow a conservative route, avoiding neurointensive care.

\section{Surgical management of haemorrhagic stroke}

Intracranial haemorrhage (ICH) in older people is often the result of long-standing underlying pathological vascular disease. A patient, therefore, has the intracranial pressure effects and focal neurological deficits associated with the ICH in addition to the systemic co-morbidities of cardiac, peripheral vascular disease, and often anti-thrombotic medication use. Furthermore, secondary haemorrhage is always a concern.

The STICH I and II trials did not demonstrate any overall benefit from early surgery compared with initial conservative management for supratentorial (including lobar) ICH. $[22,23]$. The median patient age for STICH I was 62 years (IQR 52-70) and 65 years (IQR 55-74) for STICH II. The inference from these studies is that surgeons are already appropriately undertaking selective, targeted surgery to the patients that are most likely to benefit from it. When there is equipoise about whether to operate, these studies suggest 
that it is reasonable to manage the patient conservatively in the first instance and then re-assess.

More recently, there has been a move towards minimallyinvasive surgery (MIS) for ICH, with the potential benefits of being less traumatic, quicker, and more focused than a craniotomy. However, controversy exists concerning the widespread clinical application of such techniques. A recent meta-analysis (2018) on MIS for hypertensive ICH (the most common cause of spontaneous ICH), reported a positive effect on patient prognosis (using GOS) compared to both craniotomy and conservative treatment [37]. Mortality rates were lower for MIS compared to conservative treatment and post-operative re-bleeding rates were lower for MIS compared to craniotomy. As this review only included eight randomised controlled trials and most studies also excluded patients that were $>80$ years old, more high-quality studies and in a wider population of older patients are needed before firm conclusions can be drawn. This is particularly important as $29-34 \%$ of patients diagnosed with an ICH are $\geq 80$ years old, and this patient group has significantly higher rates of in-hospital mortality and unfavourable outcome [3, 35].

Scaggiante also published a meta-analysis of MIS in 2018, assessing 15 RCTs that mainly deployed endoscopic and/or stereotactic thrombolytic techniques [32]. This consolidated the finding that MIS improved outcome compared to both craniotomy and conservative treatment. Different MIS techniques (endoscopy and stereotactic thrombolysis) both showed significant improvements, but these techniques have not been compared directly. Earlier MIS evacuation of an ICH appeared to be associated with a better chance of achieving functional independence. Conversely, the final results of the MISTIE 3 (Minimially Invasive Surgery with Thrombolysis in ICH Evacuation) trial showed that aspiration and thrombolytic irrigation of an ICH with alteplase via an image directed catheter did not improve functional outcomes compared with standard care for large ICHs [14]. A modest survival benefit was identified. This trial only included patients aged 52-71 years old, so caution must be exercised in applying the conclusions to older patients.

\section{Frailty scales}

The comprehensive geriatric assessment (CGA) is an established tool used to assess the needs of older people and implement investigations and treatments to improve long-term outcomes. The use of this tool has been shown to improve post-operative outcomes in older patients undergoing elective surgery across specialties [28]. It is, therefore, clear that older people have different needs, which when identified and addressed can improve outcomes. The CGA is a robust but cumbersome assessment including 6 different domains (medical, mental health, functional capacity, social circumstances, environment, and risk score). Identification of simpler tools, possibly even specific to neurosurgery, are necessary. Understanding what is meant by frailty is also important, as, although there is some cross-over with disability and co-morbidity, it actually refers to a physiological state of increased vulnerability to stressors due to decreased physiological reserve [12].

A frailty score based on the assessment of data from 27,098 cranial neurosurgical cases has been reported, where higher scores had good sensitivity and specificity for predicting increased 30-day mortality [39]. The score considered 19 predictors of mortality, with the most significant including ascites, ventilator dependency and renal failure, alongside more common problems such as anti-hypertensive medication use, high white cell count and low body mass index. The disadvantages are that this score still contains a large number of variables and requires prospective evaluation. Tools such as the modified Frailty Index (mFI), that have already been well validated in surgical populations, may be more useful [11, 40]. Youngerman reviewed 9149 patients with brain tumours and found increased higher mFI scores were associated with increased mortality, severe neurological complications and prolonged length of stay [46]. Combined assessment of mFI, age and ASA (American Society of Anaesthesiologists) classification gave the best predictive ability on overall outcome. A simpler scale, the clinical frailty scale, has also been applied in neurosurgical patients, predicting prognosis in operated CSDH [29, 34]. Frailty has also been correlated with poor outcome in older patients with aneurysmal SAH, however this was a very simplistic assessment of frailty based on haemoglobin, albumin and BMI [47].

Standardised assessments of frailty are needed, with validation in large cohorts of neurosurgical patients across a range of pathologies. This may then support the development of prognostication tools and aid clinical decisionmaking and family discussions.

\section{Conclusion}

At what point we consider a person to be "elderly" is shifting, as patients are fitter and more independent until later in life. There are also neurosurgical advances which may preferentially benefit older patients with multiple co-morbidities such as endovascular coiling and MIS surgery for ICH. Patients aged over 65 years of age certainly do benefit from acute neurosurgical interventions, but there is more uncertainty and risk for those aged over 80 . An age cut-off itself may not be helpful, but rather frailty should be considered instead, and we advocate improved reporting of this metric in future trials and studies. Most importantly, given the lack of robust evidence, determining patient eligibility for 
intervention is often left to the treating clinician. Whilst it is always possible to intervene, it is not always appropriate and a significantly lower likelihood of maintaining the quality of life in older patients for the conditions discussed must be recognised. On the other hand, a therapeutic nihilism may be deterministic and thwart development of improved clinical practice in this group of patients.

\section{Compliance with ethical standards}

Conflicts of interest On behalf of all authors, the corresponding author states that there is no conflict of interest.

\section{References}

1. Alford EN, Rotman LE, Erwood MS, Oster RA, Davis MC, Pittman HBC, Zeiger HE, Fisher WS (2019) Development of the Subdural Hematoma in the Elderly (SHE) score to predict mortality. J Neurosurg. https://doi.org/10.3171/2019.1.JNS182895

2. Andrews PJD, Sinclair HL, Rodriguez A, Harris BA, Battison CG, Rhodes JKJ, Murray GD (2015) Hypothermia for Intracranial Hypertension after Traumatic Brain Injury. N Engl J Med 373:2403-2412

3. Beekman R, Chu S, Sommaruga S, King Z, Kamel H, Matouk C, Hwang D, Sheth K, Falcone G (2018) Spontaneous intracerebral hemorrhage in the elderly population (S10.006). Neurology 90:S10.006

4. Brennan PM, Kolias AG, Joannides AJ, Shapey J, Marcus HJ, Gregson BA, Grover PJ, Hutchinson PJ, Coulter IC (2017) The management and outcome for patients with chronic subdural hematoma: a prospective, multicenter, observational cohort study in the United Kingdom. J Neurosurg 127:732-739

5. Chesnut RM, Temkin N, Carney N, Dikmen S, Rondina C, Videtta W, Petroni G, Lujan S, Pridgeon J, Barber J, Machamer J, Chaddock K, Celix JM, Cherner M, Hendrix T, Global Neurotrauma Research G (2012) A trial of intracranial-pressure monitoring in traumatic brain injury. N Engl J Med 367:2471-2481

6. Chibbaro S, Di Rocco F, Makiese O, Mirone G, Marsella M, Lukaszewicz AC, Vicaut E, Turner B, Hamdi S, Spiriev T, Di Emidio P, Pirracchio R, Payen D, George B, Bresson D (2010) Neurosurgery and elderly: analysis through the years. Neurosurg Rev 34:229-234

7. Cooper DJ, Rosenfeld JV, Murray L, Arabi YM, Davies AR, D’Urso P, Kossmann T, Ponsford J, Seppelt I, Reilly P, Wolfe R (2011) Decompressive craniectomy in diffuse traumatic brain injury. N Engl J Med 364:1493-1502

8. Edlmann E (2017) Pathophysiology of chronic subdural haematoma: inflammation, angiogenesis and implications for pharmacotherapy. J Neuroinflamm 14:108

9. Emich S, Richling B, McCoy MR, Al-Schameri RA, Ling F, Sun L, Wang Y, Hitzl W (2014) The efficacy of dexamethasone on reduction in the reoperation rate of chronic subdural hematomathe DRESH study: straightforward study protocol for a randomized controlled trial. Trials 15:6

10. Evans LR, Jones J, Lee HQ, Ganter D, Jaison A, Matthew J, Fitzgerald MC, Rosenfeld JV, Hunn MK, Tee JW (2019) Prognosis of acute subdural hematoma in the elderly: a systematic review. J Neurotrauma 36:517-522

11. Farhat JS, Velanovich V, Falvo AJ, Horst HM, Swartz A, Patton JH Jr, Rubinfeld IS (2012) Are the frail destined to fail? Frailty index as predictor of surgical morbidity and mortality in the elderly. J Trauma Acute Care Surg 72:1526-1530 discussion 1530-1521

12. Fried LP, Ferrucci L, Darer J, Williamson JD, Anderson G (2004) Untangling the concepts of disability, frailty, and comorbidity: implications for improved targeting and care. J Gerontol Ser A Biol Sci Med Sci 59:255-263

13. Gardner RC, Dams-O'Connor K, Morrissey MR, Manley GT (2018) Geriatric traumatic brain injury: epidemiology, outcomes, knowledge gaps, and future directions. J Neurotrauma 35:889-906

14. Hanley DF, Thompson RE, Rosenblum M, Yenokyan G, Lane K, McBee N, Mayo SW, Bistran-Hall AJ, Gandhi D, Mould WA, Ullman N, Ali H, Carhuapoma JR, Kase CS, Lees KR, Dawson J, Wilson A, Betz JF, Sugar EA, Hao Y, Avadhani R, Caron J-L, Harrigan MR, Carlson AP, Bulters D, LeDoux D, Huang J, Cobb C, Gupta G, Kitagawa R, Chicoine MR, Patel H, Dodd R, Camarata PJ, Wolfe S, Stadnik A, Money PL, Mitchell P, Sarabia R, Harnof S, Barzo P, Unterberg A, Teitelbaum JS, Wang W, Anderson CS, Mendelow AD, Gregson B, Janis S, Vespa P, Ziai W, Zuccarello M, Awad IA, Abdul-Rahim A, Abou-Hamden A, Abraham M, Ahmed A, Alba CA, Aldrich EF, Altschul D, Amin-Hanjani S, Anderson D, Ansari S, Antezana D, Ardelt A, Arikan F, Baguena M, Baker A, Barrer SJ, Becker KJ, Bergman T, Boström A, Braun J, Brindley P, Broaddus WC, Brown R, Buki A, Cao B, Cao Y, Carrion-Penagos J, Chalela J, Chang T, Chorro IM, Chowdhry S, Corral L, Csiba L, Davies J, Díaz AT, Derdeyn CP, Diringer M, Dlugash R, Ecker R, Economas T, Enriquez P, Ezer E, Fan Y, Feng H, Franz D, Freeman WD, Fusco M, Galicich W, Gelea ML, Goldstein J, Gonzalez AC, Grabarits C, Greenberg S, Gress D, Gu E, Hall C, Hernandez FM, Hoesch R, Hoh BL, Houser J, Hu R, Huang Y, Hussain MA, Insinga S, Jadhav A, Jaffe J, Jahromi BS, Jallo J, James M, James RF, Jankowitz B, Jeon E, Jichici D, Jonczak K, Jonker B, Karlen N, Keric N, Kerz T, Knopman J, Koenig C, Krishnamurthy S, Kumar A, Kureshi I, Laidlaw J, Lakhanpal A, Latorre JG, Leifer D, Leiphart J, Lenington S, Li Y, Lopez G, Lovick D, Lumenta C, Luo J, Maas MB, MacDonald J, MacKenzie L, Madan V, Majkowski R, Major O, Malhorta R, Malkoff M, Mangat H, Maswadeh A, Matouk C, McArthur K, McCaul S, Medow J, Mezey G, Mighty J, Miller D, Mohan KK, Muir K, Muñoz L, Nakaji P, Nee A, Nekoovaght-Tak S, Nyquist P, O'Kane R, Okasha M, O'Kelly C, Ostapkovich N, Pandey A, Parry-Jones A, Perla KR, Pollack A, Polster S, Pouratian N, Quinn T, Rajajee V, Reddy K, Rehman M, Reimer R, Rincon F, Rybinnik I, Sanchez B, Sansing L, Schneck M, Schuerer L, Schul D, Schweitzer J, Seder DB, Seyfried D, Sheth K, Spiotta A, Stechison M, Szabo K, Tamayo G, Tanczos K, Taussky P, Terry J, Testai F, Thomas K, Thompson CB, Thompson G, Torner JC, Tran H, Tucker K, Ungar L, Varelas P, Vargas NM, Vatter H, Venkatasubramanian C, Vermillion K, Vollmer D, Wang Y, Wang Y, Wen J, Whitworth LT, Willis B, Wrencher M, Wright SE, Xu Y, Yanase L, Yi X, Yu Z, Zomorodi A (2019) Efficacy and safety of minimally invasive surgery with thrombolysis in intracerebral haemorrhage evacuation (MISTIE III): a randomised, controlled, open-label, blinded endpoint phase 3 trial. The Lancet 393:1021-1032

15. Henaux PL, Le Reste PJ, Laviolle B, Morandi X (2017) Steroids in chronic subdural hematomas (SUCRE trial): study protocol for a randomized controlled trial. Trials 18:252

16. Hutchinson PJ, Kolias AG, Timofeev IS, Corteen EA, Czosnyka M, Timothy J, Anderson I, Bulters DO, Belli A, Eynon CA, Wadley J, Mendelow AD, Mitchell PM, Wilson MH, Critchley G, Sahuquillo J, Unterberg A, Servadei F, Teasdale GM, Pickard JD, Menon DK, Murray GD, Kirkpatrick PJ (2016) Trial of decompressive craniectomy for traumatic intracranial hypertension. $\mathrm{N}$ Engl J Med 375:1119-1130

17. International Mission for Prognosis and Analysis of Clinical Trials in TBI (2020) IMPACT Prognostic calculator. In, https://www. tbi-impact.org/?p=impact/calc. Accessed 4 Apr 2020 
18. Koffijberg H, Buskens E, Rinkel GJE (2011) Aneurysm occlusion in elderly patients with aneurysmal subarachnoid haemorrhage: a cost-utility analysis. J Neurol Neurosurg Psychiatry 82:718-727

19. Kolias AG, Edlmann E, Thelin EP, Bulters D, Holton P, Suttner N, Owusu-Agyemang K, Al-Tamimi YZ, Gatt D, Thomson S, Anderson IA, Richards O, Whitfield P, Gherle M, Caldwell K, Davis-Wilkie C, Tarantino S, Barton G, Marcus HJ, Chari A, Brennan P, Belli A, Bond S, Turner C, Whitehead L, Wilkinson I, Hutchinson PJ (2018) Dexamethasone for adult patients with a symptomatic chronic subdural haematoma (Dex-CSDH) trial: study protocol for a randomised controlled trial. Trials 19:670

20. Lucke-Wold B, Turner R, Josiah D, Knotts C, Bhatia S (2016) Do age and anticoagulants affect the natural history of acute subdural hematomas? Arch Emerg Med Cri Care 1:1010

21. McDougall CG, Spetzler RF, Zabramski JM, Partovi S, Hills NK, Nakaji P, Albuquerque FC (2012) The barrow ruptured aneurysm. Trial 116:135

22. Mendelow AD, Gregson BA, Fernandes HM, Murray GD, Teasdale GM, Hope DT, Karimi A, Shaw MD, Barer DH (2005) Early surgery versus initial conservative treatment in patients with spontaneous supratentorial intracerebral haematomas in the International Surgical Trial in Intracerebral Haemorrhage (STICH): a randomised trial. Lancet 365:387-397

23. Mendelow AD, Gregson BA, Rowan EN, Murray GD, Gholkar A, Mitchell PM (2013) Early surgery versus initial conservative treatment in patients with spontaneous supratentorial lobar intracerebral haematomas (STICH II): a randomised trial. Lancet 382:397-408

24. Miah IP, Holl DC, Peul WC, Walchenbach R, Kruyt N, de Laat K, Koot RW, Volovici V, Dirven CMF, van Kooten F, Kho KH, den Hertog HM, van der Naalt J, Jacobs B, Groen RJM, Lingsma HF, Dammers R, Jellema K, van der Gaag NA (2018) Dexamethasone therapy versus surgery for chronic subdural haematoma (DECSA trial): study protocol for a randomised controlled trial. Trials 19:575

25. Molyneux A (2002) International Subarachnoid Aneurysm Trial (ISAT) of neurosurgical clipping versus endovascular coiling in 2143 patients with ruptured intracranial aneurysms: a randomised trial. The Lancet 360:1267-1274

26. National Institute for Health and Clinical Excellence (NICE) (2014) Head injury: assessment and early management clinical guidelines [CG176]. In:

27. Nieuwkamp DJ, Rinkel GJE, Silva R, Greebe P, Schokking DA, Ferro JM (2006) Subarachnoid haemorrhage in patients \%3e or = 75 years: clinical course, treatment and outcome. J Neurol Neurosurg Psychiatry 77:933-937

28. Partridge JS, Harari D, Martin FC, Dhesi JK (2014) The impact of pre-operative comprehensive geriatric assessment on postoperative outcomes in older patients undergoing scheduled surgery: a systematic review. Anaesthesia 69(Suppl 1):8-16

29. Rockwood K, Song X, MacKnight C, Bergman H, Hogan DB, McDowell I, Mitnitski A (2005) A global clinical measure of fitness and frailty in elderly people. CMAJ Canad Med Assoc J journal de l'Association medicale canadienne 173:489-495

30. Ryttlefors M, Enblad P, Kerr RSC, Molyneux A (2008) International subarachnoid aneurysm trial of neurosurgical clipping versus endovascular coiling. Stroke 39:2720-2726

31. Santarius T, Kirkpatrick PJ, Ganesan D, Chia HL, Jalloh I, Smielewski P, Richards HK, Marcus H, Parker RA, Price SJ,
Kirollos RW, Pickard JD, Hutchinson PJ (2009) Use of drains versus no drains after burr-hole evacuation of chronic subdural haematoma: a randomised controlled trial. Lancet 374:1067-1073

32. Scaggiante J, Zhang X, Mocco J, Kellner CP (2018) Minimally invasive surgery for intracerebral hemorrhage. Stroke 49:2612-2620

33. Scholler K, Massmann M, Markl G, Kunz M, Fesl G, Bruckmann H, Pfefferkorn T, Tonn JC, Schichor C (2013) Aneurysmal subarachnoid hemorrhage in elderly patients: long-term outcome and prognostic factors in an interdisciplinary treatment approach. $\mathrm{J}$ Neurol 260:1052-1060

34. Shimizu K, Sadatomo T, Hara T, Onishi S, Yuki K, Kurisu K (2018) Importance of frailty evaluation in the prediction of the prognosis of patients with chronic subdural hematoma. Geriatrics Gerontol Int 18:1173-1176

35. Stein M, Misselwitz B, Hamann Gerhard F, Scharbrodt W, Schummer Dorothee I, Oertel Matthias F (2012) Intracerebral hemorrhage in the Very Old. Stroke 43:1126-1128

36. Sturiale CL, Brinjikji W, Murad MH, Lanzino G (2013) Endovascular treatment of intracranial aneurysms in elderly patients. Stroke 44:1897-1902

37. Tang Y, Yin F, Fu D, Gao X, Lv Z, Li X (2018) Efficacy and safety of minimal invasive surgery treatment in hypertensive intracerebral hemorrhage: a systematic review and meta-analysis. BMC Neurol 18:136

38. The MRC CRASH trial collaborators (2020) Head Injury Prognosis, CRASH. In, https://www.crash.lshtm.ac.uk/Risk\%20calculat or/index.html. Accessed 4 Apr 2020

39. Tomlinson SB, Piper K, Kimmell KT, Vates GE (2017) Preoperative frailty score for 30-day morbidity and mortality after cranial neurosurgery. World Neurosurg 107:959-965

40. Wahl TS, Graham LA, Hawn MT, Richman J, Hollis RH, Jones CE, Copeland LA, Burns EA, Itani KM, Morris MS (2017) Association of the modified frailty index with 30-day surgical readmission. JAMA Surg 152:749-757

41. Whitehouse KJ, Jeyaretna DS, Enki DG, Whitfield PC (2016) Head injury in the elderly: what are the outcomes of neurosurgical care? World Neurosurg 94:493-500

42. Whitehouse KJ, Jeyaretna DS, Wright A, Whitfield PC (2016) Neurosurgical care in the elderly: increasing demands necessitate future healthcare planning. World Neurosurg 87:446-454

43. Wiebers DO (2003) Unruptured intracranial aneurysms: natural history, clinical outcome, and risks of surgical and endovascular treatment. The Lancet 362:103-110

44. Won S-Y, Dubinski D, Brawanski N, Strzelczyk A, Seifert V, Freiman TM, Konczalla J (2017) Significant increase in acute subdural hematoma in octo- and nonagenarians: surgical treatment, functional outcome, and predictors in this patient cohort. Neurosurg Focus 43:E10

45. Won S-Y, Dubinski D, Bruder M, Cattani A, Seifert V, Konczalla J (2017) Acute subdural hematoma in patients on oral anticoagulant therapy: management and outcome. Neurosurg Focus 43:E12

46. Youngerman BE, Neugut AI, Yang J, Hershman DL, Wright JD, Bruce JN (2018) The modified frailty index and 30-day adverse events in oncologic neurosurgery. J Neurooncol 136:197-206

47. Yue Q, Liu Y, Leng B, Xu B, Gu Y, Chen L, Zhu W, Mao Y (2016) A prognostic model for early post-treatment outcome of elderly patients with aneurysmal subarachnoid hemorrhage. World Neurosurg 95:253-261 